\title{
Design of Solar Powered Vaccine Backpack
}

\author{
Muhammad Haikal Satria ${ }^{1, *}$ Ariep Jaenul ${ }^{1}$ Adhes Gamayel ${ }^{1}$ \\ ${ }^{1}$ Dept. of Electrical Engineering, Faculty of Engineeing and Computer Science, Jakarta Global University \\ ${ }^{*}$ Corresponding author. Email: haikal@jgu.ac.id
}

\begin{abstract}
Covid-19 Pandemic effects rural and indigenous area that is hard and far to reach by vehicles. Most of the medical deliveries to this remote area is done on foot and taken days to reach destination. Vaccine consistency is affected by their ambient temperature and duration of exposure to adverse temperature in their deliveries. In this research, the development of a reliable personnel backpack that provides self-sustainable energy for vaccine cold storage is proposed. This vaccine storage should support the ambient and stable temperature with at least between $2^{\circ}-8^{\circ}$ Celsius. The problem on the transportation infrastructure in rural and remote area need to be addressed. Most of delivery is done by foot through the jungle or footpath that cannot be crossed by vehicles. Multi modal of power source is needed to accommodate the lack of electricity along the route in rural and remote area. Ergonomics and lightweight are also important for the personnel to bring the vaccine.
\end{abstract}

Keywords: vaccine carrier, cold storage chain, solar

\section{INTRODUCTION}

Current Covid-19 epidemic has worldwide impact with more than 9 million cases where 49000 cases are in Indonesia. National Research institute and bio pharmacies companies around the world are competing to find as so called the only solution to end the pandemic, vaccine for Covid-19. As the vaccine research is racing, the distribution of vaccine itself, especially in rural and remote area is having bigger challenge.

The successful vaccination program requires high immunization coverage in large population. The vaccine market is reaching developing countries and remote area due to the efforts of global immunization programmes. [1] As encouraging at it seems, the increase of immunization programs and the new vaccines introduction is putting in the problems on delivery systems that does not changed in decades. Unfortunately, only $31 \%$ of vaccines are stockouts and $55 \%$ of vaccines deliveries does not follow cold chain recommendation form WHO [2]. This is because of neglected and lack of basic vaccine handling policies, standards and quality management practices in vaccine cold chain. In 2014 alone, WHO estimated $67 \%$ vaccine deliveries are prone to be damaged and wasted [3].

Instability of vaccines often emerges as a key challenge during clinical development as well as commercial distribution. A lack of infrastructure, cold chain equipment particularly in developing countries, can lead to $75-100 \%$ of vaccines being exposed to during dissemination [4]. One of these challenges is that live, attenuated vaccines are more heat sensitive to potency loss during storage and distribution [5,8]. Most of the vaccines is made by weakening, attenuating of infectious viruses and/or bacteria that can be replicated in vivo. In terms of stability, these attenuated versions of viruses and/or bacteria should be lyophilized before administered by parental injection, nasally or orally.

The administration of vaccines through rural and remote area are prone to this challenge. Energy resources limitation and lack of transportation infrastructure are some obstacles of delivering vaccine to the rural area. Improperly maintenance or outdated cold storage equipment, lack of compliance on cold chain procedures and poor monitoring are some of the direct consequences in rural and remote area, especially in hot climate $[6,7]$.

\section{VACCINE COLD CHAIN}

The common practice to deliver vaccine and preserve the temperature is by using box filled with ice pack. Also, if the area has facilities such as freezer and refrigerator then they manage to store the vaccine in that storage. Unfortunately, both practices, even with 
refrigerator or freezer compartments of the householdgrade combination storage units do not perform as well as recommended by WHO cold chain practice [9]. Research in one of developed Europe countries, shows that only one-third of the refrigerators fulfilled the cold chain requirements, and more than $15 \%$ threatening vaccine potency [10]. There is a gap of cold chain equipment providing a promising avenue for new technological research $[6,11]$.

This research proposed reliable vaccine storage to support the cold chain especially in remote and rural area. This vaccine storage should support the ambient and stable temperature with at least between $2^{\circ}-8^{\circ}$ Celsius. The problem on the transportation infrastructure in rural and remote area need to be addressed. Most of delivery is done by foot through the jungle or footpath that cannot be crossed by vehicles. The reliable vaccine storage should be carried easily by healthcare personnel. Ergonomics and lightweight are also important for the personnel to bring the vaccine.

The proposed vaccine storage should also accommodate sufficient space for air to circulate around them. The compartment should accommodate to bring any vaccine vial size with order of vial amount. The flow of air around the vaccine vials should equally distributed within the compartment [16]. This is to minimize the risk of warm air exposed when the storage is open and to prevent the defrost $[17,18]$. The right shape of compartment is needed for the vaccine to be stable and air equally distributed.

Multi modal of power source is needed to accommodate the lack of electricity along the route in rural and remote area. The design of solar powered TEC has been proposed by [19] for the deserts remote parts. It could be one of the renewable energy modalities of the proposed portable vaccine. Several parameters such as High electrical conductivity, low thermal conductivity, and a high Seebeck coefficient are desirable for thermoelectric materials. Therefore, knowledge of the relation between electrical conductivity and thermal conductivity is essential to improve the efficiency of Solar generated power on TEC properties [20,21]. The utilization of renewable energy as alternative source could coop with the challenge in the energy infrastructure.

The personnel spend whole day to bring the vaccine storage backpack that may make them suffer from musculoskeletal disorders due to carrying nonergonomic and through the obstacle roads. The vaccine storage has weight that could give uneven spinal loading and lead this to injuries in the long usage [22,23]. The ergonomic parameters and the shape of backpack should be considered to prevent spinal disorders.

The personnel vaccine storage should also be equipped with remote monitoring to monitor the temperature and condition of the compartment. Method of communication protocol for the rural and remote area has been explored by $[24,25]$ by using mesh protocol network with large coverage rural area. The usage of multimodal communication that can be automatically handover could solve the problem of telecommunication infrastructure in rural area [26].

\section{BACKPACK DESIGN}

The contexts of vaccine storage were divided into 3D design model as the scenario for personnel backpack and also the selection of performance and characteristic of each module. As shown in figure 1, conceptual isometric view of the proposed vaccine backpack is integrated with multiple sources of power. The current stage of development, all values for every associate performance and characteristic are defined into the functional bound based on the quality metrics. This 3D design will be subjected for the thermodynamic and Multiphysics analysis for the next steps.

Vaccine is distributed to remote areas that are still not reached by two-wheeled and four-wheeled vehicles. Usually during this distribution, the vaccine can be damaged or exposed. To overcome this, the solution we can offer is to use a cooler box that is combined with the backpack / backpack function, so that the delivery will remain safe and secure. The cooler box is also equipped with GPS to be able to find out the position and path when the delivery is made. In addition, it is also equipped with a solar panel that functions to charge what the cooler box needs

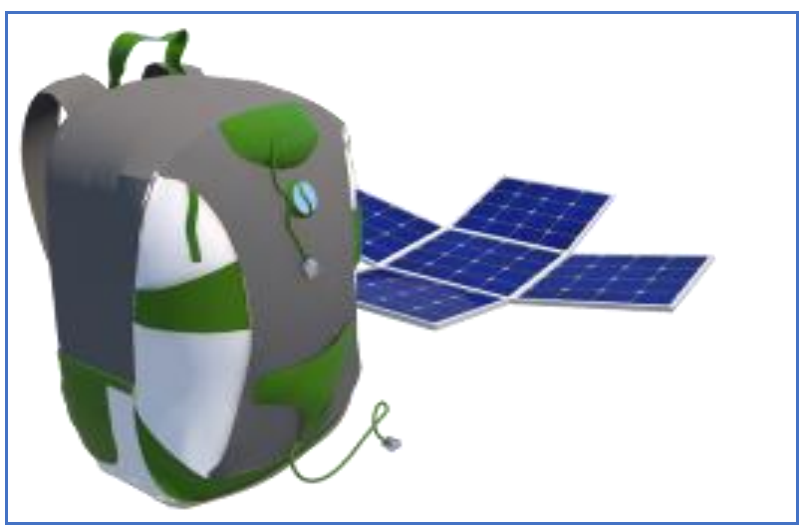

Figure 1 Conceptual isometric view of the proposed vaccine backpack.

Several solutions were made to assist in the distribution of vaccines and to ensure that there is no vaccine being wasted. The solutions we offer include in the process of delivering the vaccine, where the location is tracked and the journey process will also be recorded. Stability of the vaccine temperature and the low energy usage should be considered within the design. 


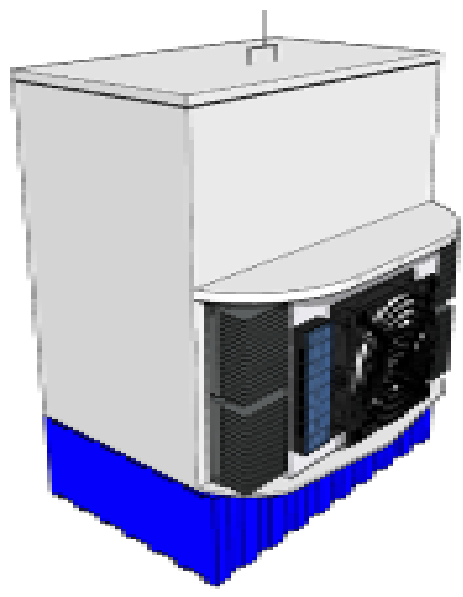

(a)

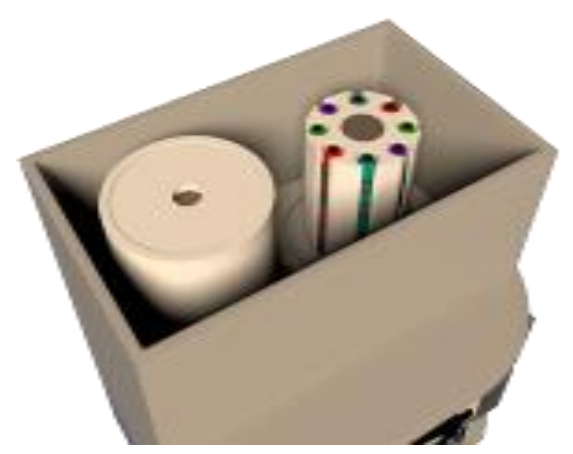

(b)

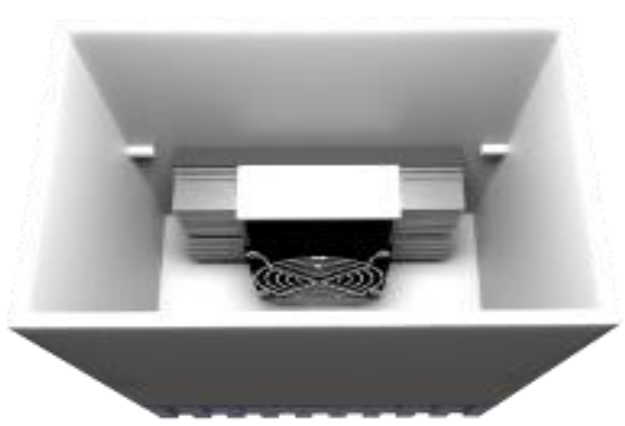

(c)

Figure 2 Cross-section design from inner part of vaccine backpack. (a) Outer layer of cold storage box (b) Vaccine vial placement and air flow steering parts (c)

Figure 2 shows the cross-section design from inner part of the backpack. The thermoelectric cooling (TEC) has been widely used for the compact, portable, low voltage and lightweight cooling device. TEC could be the solution for cooling component of our proposed portable vaccine storage. The COP is the ratio between the refrigeration load and electrical power consumption, where the calculation was based on equation below
$\operatorname{COP}=\frac{Q^{T}}{W_{e}}$

Where $Q^{T}$ is the total rate of heat gain inside the storage, and $W_{e}$ is the power consumed by the TEC unit.

However, there are several challenges such as extremely low coefficient of performance (COP) than compression system [12]. Figure 2.c shows additional heat-sink arrangement that could increase the TEC effectiveness. Configuration of heat sink increase the COP by finding optimum model of heat capacity rate of cooling fluid in the heat sink $[13,14]$. Several methods to modified mini-channel heat sinks and array configuration of TEC has also been proposed to increase the COP [15]. The best configuration array TEC and right shape of heat sink are needed to achieve the stable ambient temperature of portable vaccine storage.

As in Tabel 1, the proposed specification of vaccine backpack should generate enough power to fulfil the standards of cooling storage temperature of vaccine. Recommended storage for most of the vaccines are between $2^{\circ}-8^{\circ}$ Celsius with the shelf lives for more than 1 year. The vaccines life could be reduced to less than hours when expose to heat within certain duration.

Table 1. Proposed specification of vaccine backpack

\begin{tabular}{|l|r|}
\hline \multicolumn{1}{|c|}{ Specifications } & Descriptions \\
\hline Cooling Power QcMax (W) & 7 Watt \\
\hline Running Current & 2 Ampere \\
\hline Nominal Voltage & 5 Volt \\
\hline Max Voltage & 6.5 Volt \\
\hline Power Input & 10 Watt \\
\hline Operating Temperature & $2^{\circ} \mathrm{C}-10^{\circ} \mathrm{C}$ \\
\hline Weight & 2.5 Kilograms \\
\hline MTBF (fans-hrs) & 10,000 \\
\hline Performance tolerance & $\pm 10 \%$ \\
\hline
\end{tabular}

\section{CONCLUSION}

As $30 \%$ of population in developing countries are stayed in the remote area, the deliveries of the vaccine through remote area could be done correctly following the requirement of cold chain recommendation from WHO.

The current market of cold storage is still in the big and bulk box with compressor involved as cooling unit. There is no product that can be easily carried by personnel and reliable to keep the ambient temperature of vaccines. 
At large, the result of proposed backpack will give significant output for the vaccine cold chain landscape, as it solves the vaccine distribution to the remote area and reducing the wastage of vaccine deliveries. It will be useful not only within this pandemic, but also the medical deliveries in long term.

\section{REFERENCES}

[1] Bulletin of the World Health Organization 2011;89:324-325. doi:10.2471/BLT.11.030511

[2] World Health Organization. Achieving immunization targets with the comprehensive effective vaccine management (EVM) framework: WHO/UNICEF joint statement. No. WHO/IVB/16.09. World Health Organization, 2016.

[3] Lang, S., Ford, K. J., John, T., Pollard, A. J., \& McCarthy, N. D. (2014). Immunisation errors reported to a vaccine advice service: intelligence to improve practice. Quality in primary care, 22(3), 139-146.

[4] Wahid, A. A., Aswin Doekhie, Asel Sartbaeva, and JM H. van den Elsen. "ensilication improves the thermal Stability of the tuberculosis Antigen Ag85b and an Sbi-Ag85b Vaccine conjugate." Scientific reports 9, no. 1 (2019): 1-15.

[5] Kumru, O. S., Joshi, S. B., Smith, D. E., Middaugh, C. R., Prusik, T., \& Volkin, D. B. (2014). Vaccine instability in the cold chain: mechanisms, analysis and formulation strategies. Biologicals, 42(5), 237259.

[6] Wirkas, T., Toikilik, S., Miller, N., Morgan, C., \& Clements, C. J. (2007). A vaccine cold chain freezing study in PNG highlights technology needs for hot climate countries. Vaccine, 25(4), 691-697.

[7] Nelson, C. M., Wibisono, H., Purwanto, H., Mansyur, I., Moniaga, V., \& Widjaya, A. (2004). Hepatitis B vaccine freezing in the Indonesian cold chain: evidence and solutions. Bulletin of the World Health Organization, 82, 99-105.

[8] World Health Organization. Temperature sensitivity of vaccines. No. WHO/IVB/06.10. World Health Organization, 2006.

[9] Leidner, Andrew J., Helen Fisun, Sean Trimble, Paul Lucas, Cameron Noblit, and John M. Stevenson. "Evaluation of temperature stability among different types and grades of vaccine storage units: Data from continuous temperature monitoring devices." Vaccine (2020).
[10] Thielmann, Anika, Marie-Therese Puth, Christine Kersting, Johannes Porz, and Birgitta Weltermann. "Vaccine cold chain in general practices: A prospective study in 75 refrigerators (Keep Cool study)." PloS one 14, no. 11 (2019): e0224972.

[11] De Boeck, Kim, Catherine Decouttere, and Nico Vandaele. "Vaccine distribution chains in low-and middle-income countries: A literature review." Omega (2019): 102097

[12] Mirmanto, M., S. Syahrul, and Yusi Wirdan. "Experimental performances of a thermoelectric cooler box with thermoelectric position variations." Engineering Science and Technology, an International Journal 22, no. 1 (2019): 177-184.

[13] Zhu, Lin, and Jianlin Yu. "Optimization of heat sink of thermoelectric cooler using entropy generation analysis." International Journal of Thermal Sciences 118 (2017): 168-175.

[14] Qamar, Adnan, Muhammad Farooq, Muhammad Amjad, Huma Bilal, and Muhammad Moeen Sultan. "Effect of Heat Sink Configuration on the COP of Thermoelectric Vaccine Refrigerator." Journal of Faculty of Engineering \& Technology 23, no. 1 (2009): 33-43.

[15] Gökçek, Murat, and Fatih Şahin. "Experimental performance investigation of minichannel water cooled-thermoelectric refrigerator." Case Studies in Thermal Engineering 10 (2017): 54-62.

[16] Bell, Karen N., Carol JR Hogue, Claudine Manning, and Alan P. Kendal. "Risk factors for improper vaccine storage and handling in private provider offices." Pediatrics 107, no. 6 (2001): e100-e100.

[17] Weir, Erica, and Kathy Hatch. "Preventing cold chain failure: vaccine storage and handling." Cmaj 171, no. 9 (2004): 1050-1050.

[18] Gazmararian, Julie A., Natalia V. Oster, Diane C. Green, Linda Schuessler, Kelly Howell, Janona Davis, Marybeth Krovisky, and Samuel W. Warburton. "Vaccine storage practices in primary care physician offices: assessment and intervention." American journal of preventive medicine 23, no. 4 (2002): 246-253.

[19] Abdul-Wahab, Sabah A., Ali Elkamel, Ali M. AlDamkhi, A. Is' haq, Hilal S. Al-Rubai'ey, Abdulaziz K. Al-Battashi, Ali R. Al-Tamimi, Khamis H. Al-Mamari, and Muhammad U. Chutani. "Design and experimental investigation of portable solar thermoelectric refrigerator." Renewable Energy 34, no. 1 (2009): 30-34. 
[20] Elsheikh, Mohamed Hamid, Dhafer Abdulameer Shnawah, Mohd Faizul Mohd Sabri, Suhana Binti Mohd Said, Masjuki Haji Hassan, Mohamed Bashir Ali Bashir, and Mahazani Mohamad. "A review on thermoelectric renewable energy: Principle parameters that affect their performance." Renewable and sustainable energy reviews 30 (2014): 337-355.

[21] Zhao, Dongliang, and Gang Tan. "A review of thermoelectric cooling: materials, modeling and applications." Applied Thermal Engineering 66, no. 1-2 (2014): 15-24.

[22] Mirmohammadi, Seyyed Jalil, Mohammad Reza Nadri, Amir Houshang Mehrparvar, Mohammad Hossein Davari, and Mehrdad Mostaghaci. "Effect of ergonomic modification training about schoolbag on reduction of musculoskeletal complaints in primary school students." Focus on Sciences 2, no. 3 (2016).
[23] Baranak, Andrew Stephen. "Design of Backpacking Tent Accessories through the Lens of Wilderness Medicine." (2012).

[24] Satria, Muhammad Haikal, Jasmy bin Yunus, and Eko Supriyanto. "Emergency prenatal telemonitoring system in wireless mesh network." Telkomnika 12, no. 1 (2014): 123.

[25] Satria, Muhammad Haikal, Jasmy bin Yunus, and Eko Supriyanto. "802.11 s QoS Routing for Telemedicine Service." International Journal of Electrical \& Computer Engineering (2088-8708) 4, no. 2 (2014).

[26] Yew, Hoe Tung, Eko Supriyanto, Muhammad Haikal Satria, and Yuan Wen Hau. "New Vertical Handover Method to Optimize Utilization of Wireless Local Area Network in High-Speed Environment." Plos one 11, no. 11 (2016): e0165888. 